\title{
Serum 25-hydroxyvitamin D and hypertension in premenopausal and postmenopausal women: National Health and Nutrition Examination Surveys 2007-2010
}

\author{
Jung Hyun Kwak', Yun-Chul Hong ${ }^{2}$ and Yoon-Hyeong Choi ${ }^{1,3, *}$ (1) \\ 'Department of Preventive Medicine, Gachon University College of Medicine, 155 Gaetbeol-ro, Yeonsu-gu, Incheon \\ 21999, Republic of Korea: ${ }^{2}$ Department of Preventive Medicine, Seoul National University College of Medicine, \\ Seoul, Republic of Korea: ${ }^{3}$ Gachon Advanced Institute for Health Sciences and Technology, Incheon, Republic of Korea
}

Submitted 14 November 2018: Final revision received 16 May 2019: Accepted 30 July 2019: First published online 17 January 2020

\begin{abstract}
Objective: A recent meta-analysis suggested that the association between vitamin D and risk of hypertension was markedly stronger in women aged $<55$ years in observational data, while the association became null in women aged $\geq 55$ years. We therefore hypothesized that this difference in associations might potentially be caused by the change in oestrogen around menopause. Our objective was to investigate associations between vitamin D status and hypertension risk and to evaluate those associations as they may differ according to menopausal status.

Design: A cross-sectional population survey conducted by the US Centers for Disease Control and Prevention, National Center for Health Statistics.

Setting: The National Health and Nutrition Examination Surveys (NHANES) 2007-2010 formed the setting for the present study.

Participants: We analysed data from 2098 premenopausal women and 2298 postmenopausal women.

Results: After adjustment for sociodemographic, behavioural and dietary factors, higher concentrations both of serum total 25-hydroxyvitamin D (25(OH)D) and serum 25-hydroxycholecalciferol $\left(25(\mathrm{OH}) \mathrm{D}_{3}\right)$ revealed significant dose-dependent trends with lower risk of hypertension $\left(P_{\text {trend }}=0.005\right.$ and 0.014 , respectively) in premenopausal women. In those women, $25(\mathrm{OH}) \mathrm{D} \geq 50 \mathrm{nmol} / 1$ (sufficient; in contrast to deficient, vitamin $\mathrm{D}<30 \mathrm{nmol} / \mathrm{l})$ appeared to have a protective effect against hypertension $(\mathrm{OR}=0.64,95 \% \mathrm{CI} 0.39,1.02$ for total $25(\mathrm{OH}) \mathrm{D}$ and $\mathrm{OR}=0.60,95 \%$ CI $0.36,1.00$ for $\left.25(\mathrm{OH}) \mathrm{D}_{3}\right)$. Neither association with hypertension was observed in postmenopausal women.

Conclusions: Serum 25(OH)D concentrations were associated with lower risk of hypertension in premenopausal women, but not in postmenopausal women.
\end{abstract}

\author{
Keywords \\ Vitamin D \\ Hypertension \\ Menopausal status \\ Premenopausal women
}

Hypertension, one of the primary risk factors for $\mathrm{CVD}^{(1)}$, is a significant contributor to all-cause mortality in adults ${ }^{(2)}$. In order to prevent and treat this condition, it is necessary to evaluate its modifiable risk factors, including dietary and behavioural factors ${ }^{(3-6)}$.

Vitamin D is a steroid prohormone acquired through skin synthesis from sunlight and dietary intake of either foods or supplements ${ }^{(7,8)}$. There is growing evidence from experimental studies that a deficiency of vitamin $\mathrm{D}$ may be a risk factor affecting hypertension ${ }^{(9-11)}$. Although many studies have examined the association between vitamin $\mathrm{D}$ and hypertension, their epidemiological evidence has proven controversial. A recent review using meta-analysis ${ }^{(12)}$ of cross-sectional and prospective data suggested that the association between vitamin D status (measured by serum concentration of 25-hydroxyvitamin D (25(OH)D)) and the risk of hypertension was markedly stronger in women aged $<55$ years $^{(13,14)}$, while the association became null in women aged $\geq 55$ years ${ }^{(15-17)}$. That review suggested that the reason for a stronger association in younger women is still puzzling.

In this context, we hypothesized that this difference in associations between younger women and older women may be caused by the change in ovarian hormones around menopause. Several studies have suggested that the decline of oestrogen around the time of menopause has 


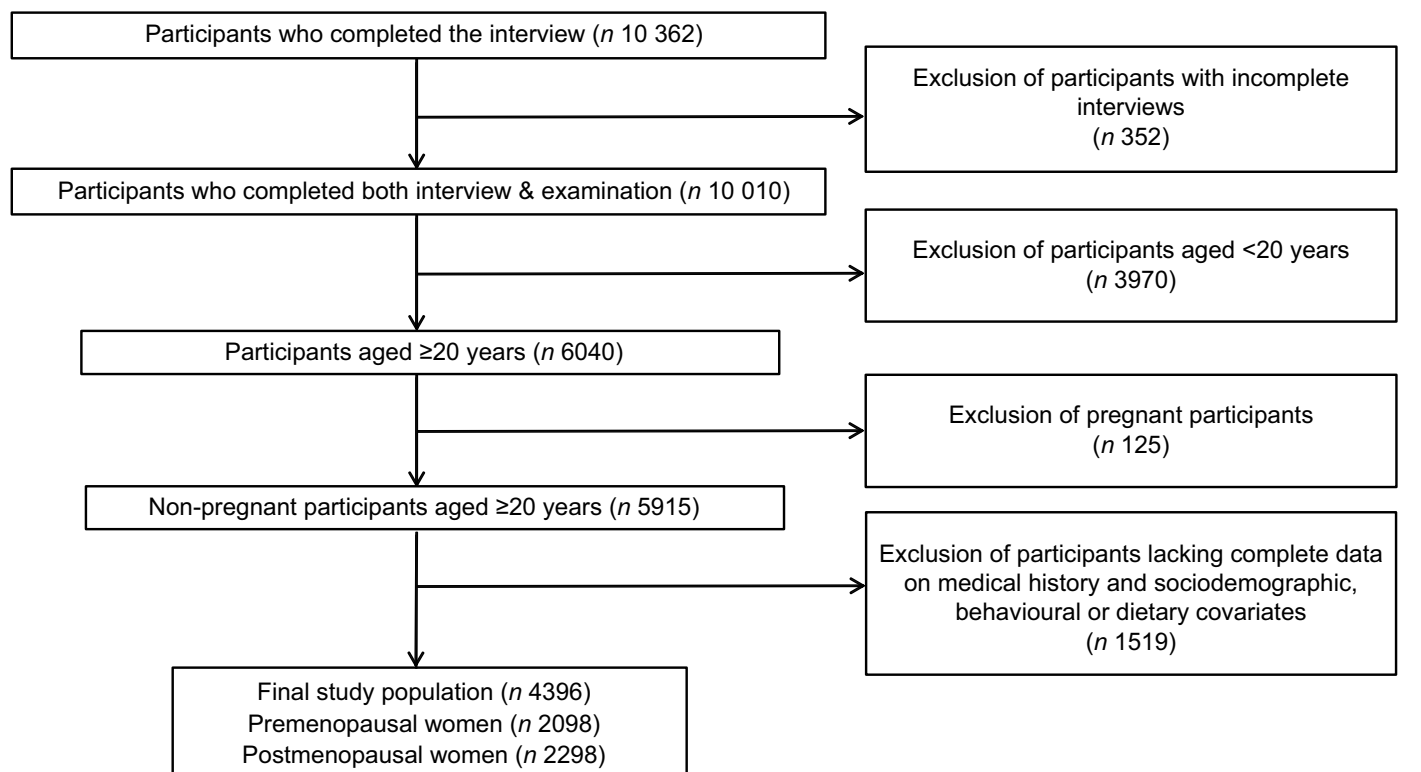

Fig. 1 Flowchart of the study population of women from the National Health and Nutrition Examination Surveys 2007-2010

various adverse effects on women's health such as increase in weight, insulin resistance and hypertension ${ }^{(18-20)}$. Maas and Franke suggested that menopausal status may promote onset of hypertension and that even premenopausal women with lower oestrogen levels caused by other hormone-related factors are at increased risk to develop hypertension or premature $\mathrm{CHD}^{(21)}$. Given the continued interest and debate on the age-specific association between vitamin $\mathrm{D}$ and hypertension in women, the current study aimed to evaluate the associations between vitamin $D$ status, measured by serum $25(\mathrm{OH}) \mathrm{D}$ concentrations, and the risk of hypertension as they may differ according to menopausal status, especially those associations in premenopausal women.

\section{Methods}

\section{Study population}

The National Health and Nutrition Examination Survey (NHANES) is a cross-sectional and population-based survey conducted to obtain health and nutritional status by the Centers for Disease Control and Prevention of the National Center for Health Statistics.

The survey uses a complex, multistage, stratified and clustered design and provides demographic, socio-economic, behavioural and dietary data on a representative sample of the civilian, non-institutionalized US population (households). Oversampled subgroups for 2007-2010 included Hispanics, non-Hispanic Blacks, low-income Whites and persons aged $\geq 80$ years. Demographic and health history information was collected through extensive household interviews, and blood and urine samples and standardized physical examinations were conducted at mobile examination centres.
The present analysis included data from four years of the NHANES surveys of 2007-2010. We did not include NHANES 1999-2006 because serum 25(OH)D concentrations were measured by a different method (i.e. RIA). Between 2007 and 2010, NHANES enrolled 10362 women. Of those, 6040 women aged $\geq 20$ years had complete data from interviews, laboratory tests and physical examinations. For the present analysis, we excluded women who were pregnant ( $n$ 125), women with missing information on serum vitamin D measures $(n 704)$ and women with missing information on hypertension ( $n$ 258) or other covariates ( $n$ 557). Hence, our statistical analysis included a total of 4396 women (2098 premenopausal women and 2298 postmenopausal women); see Fig. 1 for details.

The National Center for Health Statistics' Research Ethics Review Board reviewed and approved the study protocol (Continuation of Protocol \#2005-06) and written informed consent was obtained from all NHANES participants. NHANES is a publicly available data set. Additional details on study procedures, data documentation and questionnaires are available elsewhere ${ }^{(22,23)}$.

\section{5-Hydroxyvitamin D measurement}

Blood samples were collected from each participant at a mobile examination centre for measurement of serum $25(\mathrm{OH}) \mathrm{D}$ concentrations. The blood samples were immediately frozen at $-30^{\circ} \mathrm{C}$ and then shipped to the National Center for Environmental Health (Centers for Disease Control and Prevention, Atlanta, GA, USA) for analysis.

There are two forms of physiologically important vitamin $\mathrm{D}$ : ergocalciferol (vitamin $\mathrm{D}_{2}$ ) and cholecalciferol (vitamin $\mathrm{D}_{3}$ ). Ergocalciferol is typically obtained from fortified foods and sun-dried mushrooms, while cholecalciferol 
is synthesized in the skin from 7-dehydrocholesterol upon exposure to UVB $(290-320 \mathrm{~nm})$ and is found in oily fish (e.g. salmon, sardines and mackerel), egg yolks and fish oils (e.g. cod-liver oil) $)^{(7,8)}$.

Serum samples were measured using ultra-highperformance liquid chromatography-tandem mass spectrometry (UHPLC-MS/MS; ThermoElectron Corp., West Palm Beach, FL, USA) for the quantitative detection of 25hydroxycholecalciferol $\left(25(\mathrm{OH}) \mathrm{D}_{3}\right), 25$-hydroxyergocalciferol $\left(25(\mathrm{OH}) \mathrm{D}_{2}\right)$ and 3-epi-25-hydroxycholecalciferol (3-epi$\left.25(\mathrm{OH}) \mathrm{D}_{3}\right)^{(24,25)}$. Total $25(\mathrm{OH}) \mathrm{D}$ concentrations were summed from $25(\mathrm{OH}) \mathrm{D}_{3}$ and $25(\mathrm{OH}) \mathrm{D}_{2}$ concentrations in SI units of nmol/l; total 25(OH)D did not include 3-epi-25(OH)D $\mathrm{D}_{3}$ concentrations, a predominant form in infants under the age of 1 year ${ }^{(26)}$. The $\mathrm{CV}$ in NHANES $2007-2010$ was $3 \cdot 0-3 \cdot 8 \%$ for $25(\mathrm{OH}) \mathrm{D}_{3}$ and $3 \cdot 8-6 \cdot 5 \%$ for $25(\mathrm{OH}) \mathrm{D}_{2}$. The range of serum concentrations was within $5 \cdot 41-213 \mathrm{nmol} / 1$ for $25(\mathrm{OH}) \mathrm{D} ; 3 \cdot 02-212 \mathrm{nmol} / 1$ for $25(\mathrm{OH})$ $\mathrm{D}_{3}$; and $1.45-183 \mathrm{nmol} / 1$ for $25(\mathrm{OH}) \mathrm{D}_{2}$. For $25(\mathrm{OH}) \mathrm{D}$ levels $>1 \mathrm{ng} / \mathrm{g}$, within-set CV was $0 \cdot 1-0 \cdot 5 \%$ and between-set CV was $0 \cdot 2-0 \cdot 6 \%$; for $25(\mathrm{OH}) \mathrm{D}$ levels $<1 \mathrm{ng} / \mathrm{g}$, within-set $\mathrm{CV}$ was $0.9-2.9 \%$ and a between-set CV was within $2.0 \%{ }^{(27)}$.

In the statistical analysis, total $25(\mathrm{OH}) \mathrm{D}$ and $25(\mathrm{OH}) \mathrm{D}_{3}$ concentrations were categorized into three groups: $<30 \mathrm{nmol} / 1$ (deficient), 30-49.99 nmol/1 (insufficient) and $\geq 50 \mathrm{nmol} / 1$ (sufficient), based on cut-off points suggested by the US Institute of Medicine ${ }^{(28)}$.

\section{Hypertension}

Blood pressure measurements were taken during the NHANES examination visit. Systolic blood pressure (SBP) and diastolic blood pressure (DBP) were measured at the mobile examination centre using the Omron HEM $907 \mathrm{XL}$ apparatus (Omron Healthcare, Kyoto, Japan). After taking a rest for $5 \mathrm{~min}$ in a sitting position, blood pressure was measured at the maximum inflation level. We computed average values of three consecutive measures in SBP and DBP. Participants fulfilling one or more of the following criteria were defined as having hypertension: (i) mean $\mathrm{SBP} \geq 140 \mathrm{mmHg}$; (ii) mean $\mathrm{DBP} \geq 90 \mathrm{mmHg}$; (iii) the use of antihypertensive medication; and (iv) physician diagnosis.

\section{Menopausal status}

Menopausal status was assessed by two sequential questions: 'Have you had at least one menstrual period in the past 12 months?' If the participant answered 'no', she was then asked "What is the reason that you have not had a period in the past 12 months?' If the participant answered the second question as 'menopause' or 'hysterectomy', she was characterized as postmenopausal.

\section{Sun exposure via outdoor activities}

Sun exposure, the route of natural vitamin D synthesis, was estimated through the time spent in outdoor activities: 'How much time did you usually spend outdoors between
9 in the morning and 5 in the afternoon on the days that you worked or went to school?', to which participants answered in 'number of minutes'. Non-respondents were excluded for data analysis. Outdoor activity was grouped as <30 or $\geq 30 \mathrm{~min} / \mathrm{d}$.

\section{Covariates}

We considered participants' age, race/ethnicity, education, season of examination, physical activity, alcohol consumption, smoking status, dietary intakes (total food energy, $\mathrm{K}, \mathrm{Ca}, \mathrm{Mg}$ and $\mathrm{Na}$ ) and physical conditions (height, weight, diabetes, hormone therapy and use of birth-control pills) as potential confounders. Age was categorized as 20-29, 30-39, 40-49, 50-59, 60-69 and $\geq 70$ years. Race/ethnicity was categorized as non-Hispanic White (reference), Mexican American, non-Hispanic Black, Hispanic and other. We categorized education as less than 9th grade (reference), high-school graduate or less, and more than high-school graduate. Smoking status was categorized as never (reference), former and current smoker. Alcohol consumption was categorized as never (reference), <once/ week, 1-2 d/week, 3-4d/week and $\geq 5 \mathrm{~d} /$ week. Season of examination was classified as November to April (reference) or May to October. Diabetes was defined as self-reported physician diagnosis or current use of antihypertensive medication. Physical activity was self-reported for three levels of activity intensity (walking, moderate physical activity, vigorous physical activity). We computed each physical activity as a metabolic equivalent of task (MET) value per week; total MET scores were created by summing the weekly MET values for the three levels ${ }^{(29)}$. Information on dietary intakes (total food energy, K, Ca, $\mathrm{Mg}$ and $\mathrm{Na}$ ) was obtained through a $24 \mathrm{~h}$ dietary recall interview collected for the $24 \mathrm{~h}$ period immediately before the interview at the mobile examination centre. Hormone therapy and use of birth-control pills were self-reported and categorized as non-user or user.

\section{Statistical analysis}

Data analyses were performed using SAS survey procedure (version 9.4) in order to take account of the complex survey design and sample weights of NHANES 2007-2010. We computed 4-years sample weights from 2-years weights for 2007-2008 and 2-years weights for 2009-2010 according to NHANES analysis guideline. $P$ values are two-sided and $<0.05$ was considered statistically significant.

In descriptive analysis, we presented weighted means with their standard errors for continuous variables and weighted percentages for categorical variables; we evaluated the survey $t$ test for continuous variables and the survey (Rao-Scott) $\chi^{2}$ test for categorical variables. Serum concentrations of total $25(\mathrm{OH}) \mathrm{D}, 25(\mathrm{OH}) \mathrm{D}_{2}$ and $25(\mathrm{OH}) \mathrm{D}_{3}$ were right-skewed, so presented as geometric means.

The association between total $25(\mathrm{OH}) \mathrm{D}$ or $25(\mathrm{OH}) \mathrm{D}_{3}$ concentration and the risk of hypertension was examined using 
survey logistic regression models (PROC SURVEYLOGISTIC). We did not examine the association between $25(\mathrm{OH}) \mathrm{D}_{2}$ concentration and the risk of hypertension because many women $(22.4 \%)$ had a $25(\mathrm{OH}) \mathrm{D}_{2}$ level under the detection limit, not suitable for a reliable statistical analysis. All analyses were examined in stratified populations of pre- and postmenopausal women. In order to assess the influence of potential confounding factors, we evaluated two sequential models, Model A and Model B. Model A considered age, race/ ethnicity, education, season of examination, physical activity, alcohol consumption and smoking status. Model B considered Model A covariates plus dietary covariates, i.e. intakes of total food energy, $\mathrm{K}, \mathrm{Ca}, \mathrm{Mg}$ and $\mathrm{Na}$. Odds ratios and $95 \%$ confidence intervals were computed to estimate the association between each $25(\mathrm{OH}) \mathrm{D}$ status and the risk of hypertension by comparing each sufficient or insufficient group with the deficient group. Tests of trend were conducted for the ordinal variables of $25(\mathrm{OH}) \mathrm{D}$ in logistic regression models using integer values (1-3). In addition, we examined multiplicative interactions between serum 25(OH)D and menopause status on the risk of hypertension, computing the interaction $P$ value using Wald tests.

We also examined associations after further controlling for outdoor activity, a major source of $25(\mathrm{OH}) \mathrm{D}$, in particular $25(\mathrm{OH}) \mathrm{D}_{3}$. Information on outdoor activity was not available for participants of NHANES 2007-2008, so this further analysis was conducted using only a sub-population ( $n$ 1736) from NHANES 2009-2010.

In addition, we examined associations between $25(\mathrm{OH}) \mathrm{D}$ concentrations and blood pressure using survey linear regression models (PROC SURVEYREG) after excluding women who reported current use of antihypertensive medication ( $n$ 1522).

\section{Sensitivity analysis}

We conducted sensitivity analyses to test the robustness of our results using alternative classifications of vitamin D status: (i) as two groups of not sufficient $v$. sufficient: $<50 \mathrm{nmol} / \mathrm{l}$ and $\geq 50 \mathrm{nmol} / \mathrm{l}$; (ii) the classification suggested by the US Endocrine Society: $<50 \mathrm{nmol} / 1$ (deficient), $50-74.99 \mathrm{nmol} / 1$ (suboptimal) and $\geq 75 \mathrm{nmol} / 1$ (sufficient) ${ }^{(30)}$. Further, we ran multivariate models after further adjusting for an inflammatory marker (i.e. serum C-reactive protein concentration) or physical conditions (i.e. height, weight, diabetes, hormone therapy, use of birth-control pills) and confirmed that our results were robust to various alternative modelling approaches.

\section{Results}

\section{Descriptive statistics}

Data were available for analysis on a total of 2098 premenopausal women (mean age: 35.7 ( $\mathrm{SE} 0.32$ ) years) and 2298 postmenopausal women (mean age: $62 \cdot 3$ (SE $0 \cdot 21$ ) years).
Table 1 shows the study population's general characteristics by menopausal status. In pre- and postmenopausal women, $328(14.7 \%)$ and 1473 (58.2\%) had hypertension, respectively. The geometric means of serum total $25(\mathrm{OH}) \mathrm{D}$, $25(\mathrm{OH}) \mathrm{D}_{3}$ and $25(\mathrm{OH}) \mathrm{D}_{2}$ concentrations were 61.4, 58.6 and $1.8 \mathrm{nmol} / 1$, respectively, in premenopausal women, while the corresponding values were 66.1, 59.6 and $2.5 \mathrm{nmol} / 1$, respectively, in postmenopausal women. Total $25(\mathrm{OH}) \mathrm{D}$ and $25(\mathrm{OH}) \mathrm{D}_{2}$ concentrations were significantly higher in postmenopausal women than premenopausal women.

Table 2 shows participant characteristics by hypertension status in pre- and postmenopausal women. Among premenopausal women, participants with hypertension were likely to be older, to have lower total 25(OH)D and $25(\mathrm{OH}) \mathrm{D}_{3}$, and to have higher SBP and DBP than those without hypertension. Among postmenopausal women, participants with hypertension were likely to be older, to have lower total $25(\mathrm{OH}) \mathrm{D}$ and $25(\mathrm{OH}) \mathrm{D}_{3}$, and to have higher SBP than those without hypertension.

\section{Serum 25-bydroxyvitamin $D$ concentrations and bypertension in pre-and postmenopausal women}

Table 3 presents the associations between serum 25(OH)D concentrations and hypertension in multivariate sequential models both in pre- and postmenopausal women.

Among premenopausal women, higher concentrations both of total $25(\mathrm{OH}) \mathrm{D}$ and $25(\mathrm{OH}) \mathrm{D}_{3}$ revealed significant dose-dependent trends with a lower risk of hypertension when controlling for sociodemographic, behavioural and dietary factors (Model B, $P_{\text {trend }}=0.005$ and 0.014, respectively). Women with sufficient $(\geq 50 \mathrm{nmol} / \mathrm{l})$ total $25(\mathrm{OH}) \mathrm{D}$ and $25(\mathrm{OH}) \mathrm{D}_{3}$ had significantly lower risk of hypertension than those with deficient concentrations $(<30 \mathrm{nmol} / \mathrm{l})$ in Model A. The fully adjusted OR for hypertension comparing the sufficient $v$. the deficient groups in total 25(OH)D and 25(OH)D 3 was 0.64 (95\% CI 0.39, 1.02, $P=0.062)$ and $0.60(95 \% \mathrm{CI} 0.36,1.00, P=0.050)$, respectively. Neither the association of total $25(\mathrm{OH}) \mathrm{D}$ nor the association of $25(\mathrm{OH}) \mathrm{D}_{3}$ with hypertension in the fully adjusted models was observed among postmenopausal women ( $P_{\text {trend }}=0.364$ and $0 \cdot 155$, respectively).

We found stronger associations between $25(\mathrm{OH}) \mathrm{D}$ concentrations and lower risk of hypertension in premenopausal women than in postmenopausal women, but effect modification by menopausal status $\left(P_{\text {interaction }}=\right.$ 0.059 for total $25(\mathrm{OH}) \mathrm{D}$ and 0.114 for $\left.25(\mathrm{OH}) \mathrm{D}_{3}\right)$ did not reach statistical significance.

We conducted sensitivity analyses using alternative classifications of vitamin D status: (i) two categories (sufficient or not); and (ii) the classification suggested by the US Endocrine Society. When we compared the sufficient group $v$. the not-sufficient group (see online supplementary material, Supplemental Table S1), a significantly stronger association between total $25(\mathrm{OH}) \mathrm{D}$ concentration 
Table 1 Participant characteristics by menopausal status of the study population of women from the National Health and Nutrition Examination Surveys 2007-2010

\begin{tabular}{|c|c|c|c|c|c|}
\hline \multirow[b]{2}{*}{ Characteristic } & \multicolumn{2}{|c|}{$\begin{array}{l}\text { Premenopausal } \\
\text { women }(n 2098)\end{array}$} & \multicolumn{2}{|c|}{$\begin{array}{l}\text { Postmenopausal } \\
\text { women (n2298) }\end{array}$} & \multirow[b]{2}{*}{$P \ddagger$} \\
\hline & Mean & SE & Mean & SE & \\
\hline Total 25(OH)D $(\mathrm{nmol} / \mathrm{l}) \S$ & $61 \cdot 4$ & $1 \cdot 14$ & $66 \cdot 1$ & $1 \cdot 11$ & $<0.001$ \\
\hline $25(\mathrm{OH}) \mathrm{D}_{3}(\mathrm{nmol} / \mathrm{l}) \S$ & $58 \cdot 6$ & 1.14 & $59 \cdot 6$ & 1.02 & 0.404 \\
\hline $25(\mathrm{OH}) \mathrm{D}_{2}(\mathrm{nmol} / \mathrm{l}) \S$ & $1 \cdot 8$ & 0.03 & 2.5 & $0 \cdot 10$ & $<0.001$ \\
\hline $\mathrm{SBP}(\mathrm{mmHg})$ & $112 \cdot 4$ & 0.42 & $127 \cdot 2$ & 0.54 & $<0.001$ \\
\hline $\mathrm{DBP}(\mathrm{mmHg})$ & $68 \cdot 8$ & 0.47 & 68.6 & 0.47 & 0.605 \\
\hline Age (years) & 35.7 & 0.32 & $62 \cdot 3$ & 0.21 & $<0.001$ \\
\hline Height $(\mathrm{cm})$ & 163.5 & $0 \cdot 19$ & $161 \cdot 0$ & 0.21 & $<0.001$ \\
\hline Weight (kg) & $75 \cdot 2$ & 0.56 & $76 \cdot 2$ & 0.57 & 0.276 \\
\hline Physical activity $($ MET $\times$ h/week) & $49 \cdot 6$ & $2 \cdot 22$ & 31.6 & 1.88 & $<0.001$ \\
\hline Total food energy intake (kJ) & 7941 & 88.7 & 7104 & 77.8 & $<0.001$ \\
\hline Total food energy intake (kcal) & 1898 & $21 \cdot 2$ & 1698 & $18 \cdot 6$ & $<0.001$ \\
\hline Dietary K (mg) & 2308 & 33.2 & 2435 & $27 \cdot 9$ & 0.002 \\
\hline Dietary Ca (mg) & 896 & $15 \cdot 9$ & 846 & $12 \cdot 4$ & 0.010 \\
\hline Dietary $\mathrm{Mg}(\mathrm{mg})$ & $267 \cdot 4$ & $5 \cdot 11$ & 261.6 & 3.09 & 0.207 \\
\hline \multirow[t]{2}{*}{ Dietary $\mathrm{Na}(\mathrm{mg})$} & 3132 & $44 \cdot 4$ & 2803 & 34.9 & $<0.001$ \\
\hline & $n$ & $\%$ & $n$ & $\%$ & \\
\hline Hypertension & 328 & $14 \cdot 7$ & 1473 & $58 \cdot 2$ & $<0.001$ \\
\hline Race/ethnicity & & & & & $<0.001$ \\
\hline Non-Hispanic White & 937 & $66 \cdot 6$ & 1238 & $78 \cdot 2$ & \\
\hline Non-Hispanic Black & 372 & $12 \cdot 0$ & 397 & 9.7 & \\
\hline Mexican American & 426 & 9.4 & 361 & 4.9 & \\
\hline Other ethnicity & 363 & $12 \cdot 0$ & 302 & $7 \cdot 2$ & \\
\hline Alcohol consumption & & & & & $<0.001$ \\
\hline Never & 589 & $23 \cdot 6$ & 1107 & $40 \cdot 5$ & \\
\hline$<$ Once/week & 1005 & 47.0 & 755 & $35 \cdot 0$ & \\
\hline $1-2 \mathrm{~d} /$ week & 327 & $18 \cdot 8$ & 202 & 11.1 & \\
\hline 3-4 d/week & 117 & 7.4 & 94 & $5 \cdot 2$ & \\
\hline$\geq 5 \mathrm{~d} /$ week & 60 & $3 \cdot 1$ & 140 & $8 \cdot 2$ & \\
\hline Smoking status & & & & & $<0.001$ \\
\hline Never & 1351 & $62 \cdot 9$ & 1339 & $56 \cdot 1$ & \\
\hline Former & 248 & $14 \cdot 2$ & 619 & 29.2 & \\
\hline Current & 499 & $22 \cdot 9$ & 340 & 14.6 & \\
\hline Season of examination & & & & & 0.019 \\
\hline November to April & 985 & $40 \cdot 1$ & 990 & $35 \cdot 3$ & \\
\hline May to October & 1113 & 59.9 & 1308 & $64 \cdot 7$ & \\
\hline Education (\%) & & & & & $<0.001$ \\
\hline$<9$ th grade & 163 & 4.3 & 354 & $7 \cdot 3$ & \\
\hline$\leq$ High-school graduate & 769 & $31 \cdot 7$ & 975 & 41.0 & \\
\hline >High-school graduate & 1166 & 64.1 & 969 & $51 \cdot 7$ & \\
\hline Diabetes & 88 & 3.4 & 476 & $15 \cdot 0$ & $<0.001$ \\
\hline Outdoor $\operatorname{activity}(\geq 30 \mathrm{~min} / \mathrm{d}) \|$ & 503 & 60.5 & 139 & 23.4 & $<0.001$ \\
\hline Use of hormone therapy & 8 & 0.5 & 150 & 8.4 & $<0.001$ \\
\hline Use of birth-control pills & 265 & $15 \cdot 5$ & - & & - \\
\hline
\end{tabular}

and lower risk of hypertension was found in premenopausal women than in postmenopausal women, with a significant interaction by menopausal status in the fully adjusted model $\left(P_{\text {interaction }}=0.016\right)$. When we used a different classification by the US Endocrine Society (see online supplementary material, Supplemental Table S2), the results were consistent with those obtained using the original classification (Table 3).

\section{Outdoor activity, serum 25-hydroxyvitamin D concentrations and bypertension}

Table 4 presents the outdoor activity-adjusted associations between total $25(\mathrm{OH}) \mathrm{D}$ or $25(\mathrm{OH}) \mathrm{D}_{3}$ concentration and hypertension in a sub-population with data on outdoor activity, a major source of vitamin D. No associations were observed in either group (premenopausal women, $n 825$; postmenopausal women, $n 911$ ), unlike the significant 
Table 2 Participant characteristics by hypertension status of the study population of pre- and postmenopausal women from the National Health and Nutrition Examination Surveys 2007-2010

\begin{tabular}{|c|c|c|c|c|c|}
\hline \multirow[b]{2}{*}{ Characteristic } & \multicolumn{2}{|c|}{$\begin{array}{c}\text { No } \\
\text { hypertension }\end{array}$} & \multicolumn{2}{|c|}{ Hypertension } & \multirow[b]{2}{*}{$P \ddagger$} \\
\hline & Mean & SE & Mean & SE & \\
\hline Premenopausal women & \multicolumn{2}{|c|}{$n 1770$} & \multicolumn{2}{|c|}{$n 328$} & \\
\hline Total 25(OH)D (nmol/l)§ & $62 \cdot 6$ & 1.28 & $55 \cdot 0$ & 1.91 & 0.002 \\
\hline $25(\mathrm{OH}) \mathrm{D}_{3}(\mathrm{nmol} / \mathrm{l}) \S$ & $59 \cdot 9$ & $1 \cdot 30$ & $51 \cdot 7$ & $1 \cdot 76$ & 0.001 \\
\hline $25(\mathrm{OH}) \mathrm{D}_{2}(\mathrm{nmol} / \mathrm{l}) \S$ & 1.7 & 0.03 & 1.9 & 0.09 & 0.205 \\
\hline $\mathrm{SBP}(\mathrm{mmHg})$ & $109 \cdot 9$ & 0.38 & $126 \cdot 7$ & 0.90 & $<0.001$ \\
\hline $\mathrm{DBP}(\mathrm{mmHg})$ & $67 \cdot 3$ & 0.44 & $77 \cdot 7$ & 0.84 & $<0.001$ \\
\hline Age (years) & 34.9 & 0.34 & $40 \cdot 5$ & 0.56 & $<0.001$ \\
\hline Postmenopausal women & \multicolumn{2}{|c|}{$n 825$} & \multicolumn{2}{|c|}{$n 1473$} & \\
\hline Total 25(OH)D $(\mathrm{nmol} / \mathrm{l}) \S$ & 68.9 & 1.38 & $64 \cdot 2$ & 1.14 & $<0.001$ \\
\hline $25(\mathrm{OH}) \mathrm{D}_{3}(\mathrm{nmol} / \mathrm{l}) \S$ & $63 \cdot 5$ & 1.33 & 56.9 & 1.05 & $<0.001$ \\
\hline $25(\mathrm{OH}) \mathrm{D}_{2}(\mathrm{nmol} / \mathrm{l}) \S$ & $2 \cdot 3$ & 0.12 & 2.6 & 0.12 & 0.099 \\
\hline $\mathrm{SBP}(\mathrm{mmHg})$ & $116 \cdot 8$ & 0.58 & $134 \cdot 7$ & 0.65 & $<0.001$ \\
\hline $\mathrm{DBP}(\mathrm{mmHg})$ & 68.5 & 0.45 & $68 \cdot 8$ & 0.63 & 0.621 \\
\hline Age (years) & $57 \cdot 9$ & 0.40 & $65 \cdot 4$ & 0.30 & $<0.001$ \\
\hline
\end{tabular}

Total 25(OH)D, total 25-hydroxyvitamin D; 25(OH) $\mathrm{D}_{3}$, 25-hydroxycholecalciferol; $25(\mathrm{OH}) \mathrm{D}_{2}$, 25-hydroxyergocalciferol; SBP, systolic blood pressure; DBP, diastolic blood pressure.

$\ddagger P<0.05$ is considered significant.

$\S$ Geometric means are presented.

Table 3 Odds ratios and $95 \%$ confidence intervals for hypertension (HTN) by total 25-hydroxyvitamin D (25(OH)D) and 25hydroxycholecalciferol $\left(25(\mathrm{OH}) \mathrm{D}_{3}\right)$ concentrations in the study population of pre- and postmenopausal women from the National Health and Nutrition Examination Surveys 2007-2010

\begin{tabular}{|c|c|c|c|c|c|c|c|c|c|c|}
\hline \multirow[b]{3}{*}{ Variable } & \multicolumn{5}{|c|}{ Total 25(OH)D (nmol/l) } & \multicolumn{5}{|c|}{$25(\mathrm{OH}) \mathrm{D}_{3}(\mathrm{nmol} / \mathrm{l})$} \\
\hline & \multirow{2}{*}{$\begin{array}{l}\text { No. HTN/no. } \\
\text { participants }\end{array}$} & \multicolumn{2}{|c|}{ Model A } & \multicolumn{2}{|c|}{ Model B } & \multirow{2}{*}{$\begin{array}{c}\text { No. HTN/no. } \\
\text { participant }\end{array}$} & \multicolumn{2}{|r|}{ Model A } & \multicolumn{2}{|c|}{ Model B } \\
\hline & & OR & $95 \% \mathrm{Cl}$ & OR & $95 \% \mathrm{Cl}$ & & OR & $95 \% \mathrm{Cl}$ & OR & $95 \% \mathrm{Cl}$ \\
\hline \multicolumn{11}{|c|}{ Premenopausal women (n2098) } \\
\hline$<30$ (deficient) & $49 / 238$ & 1.00 & Ref. & 1.00 & Ref. & $64 / 292$ & 1.00 & Ref. & 1.00 & Ref. \\
\hline 30-49.99 (insufficient) & $113 / 586$ & 1.20 & $0.78,1.85$ & 1.23 & $0.79,1.92$ & $107 / 605$ & 1.03 & $0.73,1.44$ & 1.06 & $0.75,1.52$ \\
\hline$\geq 50$ (sufficient) & $166 / 1274$ & 0.59 & $0.37,0.95^{*}$ & 0.64 & $0.39,1.02 \dagger$ & $157 / 1201$ & 0.55 & $0.33,0.91^{*}$ & 0.60 & $0.36,1.00+$ \\
\hline $\bar{P}_{\text {trend }}$ & & \multicolumn{2}{|c|}{$\begin{array}{l}0.59 \\
0.002\end{array}$} & \multicolumn{2}{|c|}{$\begin{array}{l}0.005 \\
0.00,1.0<1\end{array}$} & & \multicolumn{2}{|c|}{0.005} & \multicolumn{2}{|c|}{0.014} \\
\hline \multicolumn{11}{|c|}{ Postmenopausal women ( $n 2298)$} \\
\hline$<30$ (deficient) & $142 / 195$ & 1.00 & Ref. & 1.00 & Ref. & $223 / 309$ & 1.00 & Ref. & 1.00 & Ref. \\
\hline 30-49.99 (insufficient) & $328 / 505$ & 0.67 & $0.43,1.04 \dagger$ & 0.68 & $0.44,1.04 \dagger$ & $365 / 562$ & 0.76 & $0.49,1.18$ & 0.80 & $0.51,1.25$ \\
\hline$\geq 50$ (sufficient) & $1003 / 1598$ & 0.66 & $0.43,1.01 \dagger$ & 0.71 & $0.47,1.09$ & $885 / 1427$ & 0.72 & $0.52,0.99^{*}$ & & $0.55,1.09$ \\
\hline$P_{\text {trend }}$ & & \multicolumn{2}{|c|}{0.145} & \multicolumn{2}{|c|}{0.364} & & \multicolumn{2}{|c|}{0.032} & \multicolumn{2}{|c|}{0.155} \\
\hline$P_{\text {interaction }}$ & & \multicolumn{2}{|c|}{0.053} & \multicolumn{2}{|c|}{0.059} & & \multicolumn{2}{|c|}{0.086} & \multicolumn{2}{|c|}{0.114} \\
\hline
\end{tabular}

Ref., reference category.

Model A was adjusted for age, race/ethnicity, education, season of examination, physical activity, alcohol consumption and smoking status.

Model B: Model A + further adjusted for dietary covariates (intakes of total food energy, $\mathrm{K}, \mathrm{Ca}, \mathrm{Mg}$ and $\mathrm{Na}$ ).

${ }^{*} P<0.05, \dagger P<0.1$ compared with the reference group (vitamin concentrations $\left.<30 \mathrm{nmol} / \mathrm{l}\right)$.

associations observed in Table 3. This difference was potentially due to lower power because of the smaller sample size.

\section{Serum 25-hydroxyvitamin $D$ concentrations and blood pressure in pre- and postmenopausal women}

Table 5 shows the results of the associations of serum $25(\mathrm{OH}) \mathrm{D}$ concentrations with blood pressure levels in pre- and postmenopausal women who were not taking antihypertensive medication.

Among premenopausal women ( $n$ 1824), those with sufficient total $25(\mathrm{OH}) \mathrm{D}(\geq 50 \mathrm{nmol} / \mathrm{l})$ had significantly lower SBP $(-3.08 \mathrm{mmHg}(95 \% \mathrm{CI}-5.69,-0.47 \mathrm{mmHg})$, $P=0.022)$ and lower DBP $(-2.38 \mathrm{mmHg}(95 \% \mathrm{CI}-4.84$, $0.07 \mathrm{mmHg}), P=0.057)$ than those with deficient total 25(OH)D ( $<30 \mathrm{nmol} / \mathrm{l})$. Among postmenopausal women ( $n$ 1050), those with sufficient total 25(OH)D had no significant change in SBP and DBP compared with those with deficient total $25(\mathrm{OH}) \mathrm{D}$. We found a significant interaction in DBP $\left(P_{\text {interaction }}=0.021\right)$, whereas SBP did not show a significant interaction by menopause status. The results for the associations between $25(\mathrm{OH}) \mathrm{D}_{3}$ and blood pressure levels were similar to those for total 25(OH)D. 
Table 4 Outdoor activity-adjusted odds ratios and $95 \%$ confidence intervals for hypertension by total 25-hydroxyvitamin D (25(OH)D) and 25hydroxycholecalciferol $\left(25(\mathrm{OH}) \mathrm{D}_{3}\right)$ concentrations in the study population of pre- and postmenopausal women ( $\left.n 1736\right)$ from the National Health and Nutrition Examination Surveys (NHANES) 2009-2010

\begin{tabular}{|c|c|c|c|c|c|c|c|c|c|c|}
\hline \multirow[b]{2}{*}{ Variable } & \multicolumn{2}{|r|}{ Sun§ } & \multicolumn{2}{|c|}{ Total 25(OH)D\| } & \multicolumn{2}{|c|}{$\begin{array}{l}\text { Sun + total } \\
25(\mathrm{OH}) \mathrm{D} \uparrow\end{array}$} & \multicolumn{2}{|c|}{$25(\mathrm{OH}) \mathrm{D}_{3} \|$} & \multicolumn{2}{|c|}{ Sun $+25(\mathrm{OH}) \mathrm{D}_{3}$ П } \\
\hline & OR & $95 \% \mathrm{Cl}$ & OR & $95 \% \mathrm{Cl}$ & OR & $95 \% \mathrm{Cl}$ & OR & $95 \% \mathrm{Cl}$ & OR & $95 \% \mathrm{Cl}$ \\
\hline \multicolumn{11}{|l|}{ Premenopausal women ( $n 825)$} \\
\hline \multicolumn{11}{|l|}{ Outdoor activities $(\mathrm{min} / \mathrm{d})$} \\
\hline$<30$ & 1.00 & Ref. & \multirow{2}{*}{\multicolumn{2}{|c|}{ - }} & 1.00 & Ref. & \multicolumn{2}{|c|}{ - } & 1.00 & Ref. \\
\hline$\geq 30$ & 0.73 & $0.46,1 \cdot 13$ & & & 0.74 & $0.46,1 \cdot 18$ & \multicolumn{2}{|c|}{-} & 0.73 & $0.47,1.15$ \\
\hline \multicolumn{11}{|l|}{ 25(OH)D (nmol/l) } \\
\hline$<30$ (deficient) & \multicolumn{2}{|r|}{ - } & 1.00 & Ref. & 1.00 & Ref. & 1.00 & Ref. & 1.00 & Ref. \\
\hline 30-49.99 (insufficient) & \multicolumn{2}{|r|}{-} & 1.53 & $0.68,3.42$ & 1.50 & $0.68,3.32$ & 1.26 & $0.71,2 \cdot 22$ & 1.25 & $0.72,2.15$ \\
\hline$\geq 50$ (sufficient) & \multirow{2}{*}{\multicolumn{2}{|c|}{-}} & 0.78 & $0.33,1.88$ & 0.78 & $0.46,1.18$ & 0.67 & $0.34,1.29$ & 0.67 & $0.35,1.26$ \\
\hline$P_{\text {trend }}$ & & & \multicolumn{2}{|c|}{0.144} & \multicolumn{2}{|c|}{0.140} & \multicolumn{2}{|c|}{0.080} & \multicolumn{2}{|c|}{0.074} \\
\hline \multicolumn{11}{|l|}{ Postmenopausal women ( $n$ 911) } \\
\hline \multicolumn{11}{|l|}{ Outdoor activity $(\mathrm{min} / \mathrm{d})$} \\
\hline$<30$ & 1.00 & Ref. & \multirow{2}{*}{\multicolumn{2}{|c|}{$\begin{array}{l}- \\
-\end{array}$}} & 1.00 & Ref. & \multicolumn{2}{|c|}{-} & 1.00 & Ref. \\
\hline $\begin{array}{l}\geq 30 \\
25(\mathrm{OH}) \mathrm{D}(\mathrm{nmol} / \mathrm{l})\end{array}$ & 0.56 & $0.29,1 \cdot 10 \dagger$ & & & 0.56 & $0.29,1.09 \dagger$ & \multicolumn{2}{|c|}{-} & 0.57 & $0.29,1.09 \dagger$ \\
\hline$<30$ (deficient) & \multicolumn{2}{|r|}{-} & 1.00 & Ref. & 1.00 & Ref. & 1.00 & Ref. & 1.00 & Ref. \\
\hline 30-49.99 (insufficient) & \multicolumn{2}{|c|}{-} & 0.94 & $0.42,2 \cdot 12$ & 0.97 & $0.44,2 \cdot 17$ & 0.89 & $0.40,1.99$ & 0.92 & $0.40,2 \cdot 14$ \\
\hline$\geq 50$ (sufficient) & \multirow{2}{*}{\multicolumn{2}{|c|}{-}} & 0.91 & $0.43,1.93$ & 0.94 & $0.45,1.96$ & 0.85 & $0.52,1.39$ & 0.90 & $0.56,1.44$ \\
\hline $\bar{P}_{\text {trend }}$ & & & \multicolumn{2}{|c|}{0.774} & \multicolumn{2}{|c|}{0.832} & \multicolumn{2}{|c|}{0.479} & \multicolumn{2}{|c|}{0.610} \\
\hline \multicolumn{11}{|l|}{$P_{\text {interaction }}$} \\
\hline Sun $\times$ menopause status & & 567 & & - & & 337 & & - & & 918 \\
\hline $25(\mathrm{OH}) \mathrm{D} \times$ menopause status & & - & & 18 & & 341 & & 69 & & 551 \\
\hline
\end{tabular}

Ref., reference category.

$\dagger P<0.1$ compared with the reference group (vitamin concentrations $<30 \mathrm{nmol} /$; ; outdoor activity $<30 \mathrm{~min} / \mathrm{d}$ ).

†These analyses ( $n$ 1736) used NHANES 2009-2010 because outdoor activity was not available in NHANES $2007-2008$.

§Outdoor activity models were adjusted for age, race/ethnicity, education, season of examination, physical activity, alcohol consumption, smoking status and dietary covariates.

$\|$ Serum 25(OH)D models were adjusted for age, race/ethnicity, education, season of examination, physical activity, alcohol consumption, smoking status and dietary covariates.

ๆOutdoor activity models were further adjusted for serum 25(OH)D; serum 25(OH)D models were further adjusted for outdoor activity.

Table 5 Changes in blood pressure and $95 \%$ confidence intervals by total 25-hydroxyvitamin $\mathrm{D}(25(\mathrm{OH}) \mathrm{D})$ and 25 -hydroxycholecalciferol $\left(25(\mathrm{OH}) \mathrm{D}_{3}\right)$ concentrations in the study population of pre- and postmenopausal women (after excluding participants taking antihypertensive medication) from the National Health and Nutrition Examination Surveys 2007-2010

\begin{tabular}{|c|c|c|c|c|c|c|c|c|}
\hline \multirow[b]{3}{*}{ Variable } & \multicolumn{4}{|c|}{ Total 25(OH)D (nmol/l) } & \multicolumn{4}{|c|}{$25(\mathrm{OH}) \mathrm{D}_{3}(\mathrm{nmol} / \mathrm{l})$} \\
\hline & \multicolumn{2}{|c|}{$\mathrm{SBP}(\mathrm{mmHg})$} & \multicolumn{2}{|c|}{$\mathrm{DBP}(\mathrm{mmHg})$} & \multicolumn{2}{|c|}{$\mathrm{SBP}(\mathrm{mmHg})$} & \multicolumn{2}{|c|}{$\mathrm{DBP}(\mathrm{mmHg})$} \\
\hline & $\beta$ & $95 \% \mathrm{Cl}$ & $\beta$ & $95 \% \mathrm{Cl}$ & $\beta$ & $95 \% \mathrm{Cl}$ & $\beta$ & $95 \% \mathrm{Cl}$ \\
\hline \multicolumn{9}{|c|}{ Premenopausal women ( $n$ 1824) } \\
\hline$<30$ (deficient) & 0.00 & Ref. & 0.00 & Ref. & 0.00 & Ref. & 0.00 & Ref. \\
\hline 30-49.99 (insufficient) & -1.04 & $-4 \cdot 26,2 \cdot 18$ & -0.85 & $-3.30,1.61$ & -1.01 & $-3 \cdot 33,1 \cdot 30$ & -1.00 & $-3 \cdot 17,1 \cdot 17$ \\
\hline$\geq 50$ (sufficient) & -3.08 & $-5 \cdot 69,-0.47^{*}$ & $-2 \cdot 38$ & $-4.84,0.07 \dagger$ & -2.42 & $-4.65,-0.20^{*}$ & $-2 \cdot 34$ & $-4.45,-0.23^{*}$ \\
\hline $\begin{array}{l}P_{\text {trend }} \\
\text { Postmenopausal women }\end{array}$ & \multicolumn{2}{|c|}{0.006} & \multicolumn{2}{|c|}{0.030} & \multicolumn{2}{|c|}{0.026} & \multicolumn{2}{|c|}{0.019} \\
\hline$<30$ (deficient) & 0.00 & Ref. & 0.00 & Ref. & 0.00 & Ref. & 0.00 & Ref. \\
\hline 30-49.99 (insufficient) & $-3 \cdot 14$ & $-8.37,2.08$ & -0.66 & $-3 \cdot 43,2 \cdot 12$ & -1.25 & $-5 \cdot 88,3.38$ & 0.63 & $-3.04,4.30$ \\
\hline$\geq 50$ (sufficient) & -4.24 & $-9.67,1.18$ & 0.82 & $-2.44,4.09$ & -3.39 & $-7.69,0.91$ & 1.37 & $-2.04,4.78$ \\
\hline $\bar{P}_{\text {trend }}$ & \multirow{2}{*}{\multicolumn{2}{|c|}{$\begin{array}{l}0.168 \\
0.978\end{array}$}} & \multicolumn{2}{|c|}{0.215} & \multicolumn{2}{|c|}{0.065} & \multicolumn{2}{|c|}{0.292} \\
\hline$P_{\text {interaction }}$ & & & \multicolumn{2}{|c|}{0.021} & \multicolumn{2}{|c|}{0.566} & \multicolumn{2}{|c|}{0.032} \\
\hline
\end{tabular}

SBP, systolic blood pressure; DBP, diastolic blood pressure; Ref., reference category.

These models were adjusted for age, race/ethnicity, education, season of examination, physical activity, alcohol consumption, smoking status and dietary covariates. ${ }^{\star} P<0.05, \dagger P<0.1$ compared with the reference group (vitamin $D$ concentrations $\left.<30 \mathrm{nmol} / \mathrm{l}\right)$.

\section{Discussion}

In the current study of a representative sample of US female adults, strong associations between serum 25(OH)D concentrations and the risk of hypertension were found in premenopausal women, while weak or null associations between serum $25(\mathrm{OH}) \mathrm{D}$ concentrations and the risk of hypertension were found in postmenopausal women.

To our knowledge, the present study is the first to examine the association of vitamin D status and the risk of 
hypertension according to menopausal status. After adjusting for sociodemographic, behavioural and dietary factors in data on our group of premenopausal women, higher concentrations of both total $25(\mathrm{OH}) \mathrm{D}$ and $25(\mathrm{OH}) \mathrm{D}_{3}$ revealed significant dose-dependent trends with the lower risk of hypertension; and women with sufficient vitamin $\mathrm{D}$ status (i.e. serum total $25(\mathrm{OH}) \mathrm{D}$ or $25(\mathrm{OH}) \mathrm{D}_{3}$ concentration $\geq 50 \mathrm{nmol} / \mathrm{l})$ were observed to have a protective effect against hypertension (OR $=0.64$ for total $25(\mathrm{OH}) \mathrm{D}$ and $\mathrm{OR}=0.60$ for $25(\mathrm{OH}) \mathrm{D}_{3}$; see Model $\mathrm{B}$ in Table 3). However, neither the dose-dependent trend of total $25(\mathrm{OH}) \mathrm{D}$ nor of $25(\mathrm{OH}) \mathrm{D}_{3}$ with hypertension was observed in our group of postmenopausal women. Because there has been no epidemiological study that examines the association between $25(\mathrm{OH}) \mathrm{D}$ and hypertension in a population of premenopausal women, it is impossible to compare our results with previous studies. A recent study of mixed-effect meta-analysis, performed to estimate the pooled effect of high $25(\mathrm{OH}) \mathrm{D}$ on hypertension in women $<55$ years of age, reported a pooled OR of 0.62 (95\% CI $0.44,0.87)^{(12)}$, which is comparable to our observations in premenopausal women.

There is plausible biological evidence to support the role of vitamin D in vascular health in general and the risk of high blood pressure. Several underlying mechanisms that link vitamin $\mathrm{D}$ to regulation of blood pressure have been suggested, as follows. First, vitamin D down-regulates the renin-angiotensin system, interacting with the reninangiotensin system and $\mathrm{Na}$ to influence vascular smooth muscle tone, and indirectly influencing the endothelium $^{(31)}$. Indeed, an experimental study using mice lacking the vitamin $\mathrm{D}$ receptor showed increased renin activity, development of hypertension, and more susceptibility to obstructive renal injury ${ }^{(9)}$. Second, sufficient vitamin D suppresses parathyroid hormone (PTH) in $\mathrm{Ca}$ homeostasis, while low vitamin D leads to a rise in PTH and arterial resistance leading to hypertension ${ }^{(32)}$. Third, vitamin D may improve endothelial function by reducing oxidative stress and down-regulating angiotensin II type 1 receptors $(\mathrm{AT} 1)^{(33)}$

Numerous epidemiological studies have examined the relationship between $25(\mathrm{OH}) \mathrm{D}$ and blood pressure or hypertension, but their findings have been inconsistent. Several meta-analyses based on observational studies of $25(\mathrm{OH}) \mathrm{D}^{(12)}$, intervention studies of vitamin $\mathrm{D}$ supplementation $^{(34)}$ and a mendelian randomization study of $25(\mathrm{OH}) \mathrm{D}^{(35)}$ failed to support a consistent effect of vitamin $\mathrm{D}$ status on blood pressure or hypertension. Interestingly, a recent review of $25(\mathrm{OH}) \mathrm{D}$ status and hypertension conducted by Ke et al. suggested markedly stronger evidence in younger women $<55$ years of age but no evidence in older women $\geq 55$ years of age, although the cause of these differing results is still puzzling ${ }^{(12)}$.

In the current study, the potential cause of our results, with apparent findings in premenopausal women but null findings in postmenopausal women, may be the change of oestrogen secretion around menopause ${ }^{(36)}$. In fact, previous studies revealed a negative relationship between oestrogen levels and hypertension. In animal models, Nickenig et al. found that oestradiol down-regulates the AT1 receptor gene expression in vascular smooth muscle cells ${ }^{(37)}$. Therefore, reductions in oestradiol would tend to activate the renin-angiotensin system and cause an increase in blood pressure. In particular, oestradiol up-regulates the transcription and expression of the vitamin D receptor in in vivo models ${ }^{(38)}$. It may therefore be the case that as oestrogen down-regulates the expression of AT1 receptor or up-regulates vitamin D receptor expression, those mechanisms serve to protect against hypertension in premenopausal women.

Another potential cause of our findings is the change of body weight around menopause that may cause vitamin D deficiency. Menopause is associated with obesity because women after menopause lose fat-free mass, tend to exercise less and have greater increases in fat mass ${ }^{(39)}$. Also, Wortsman et al. found that obese individuals are susceptible to low concentrations of $25(\mathrm{OH}) \mathrm{D}$ because of the decreased bioavailability of vitamin D from cutaneous and dietary sources, that is fat soluble and readily sequestered in adipose tissue ${ }^{(40)}$. Indeed, Ke et al. suggested that confounding by healthy lifestyle variables, in particular obesity, may account for the association seen between $25(\mathrm{OH}) \mathrm{D}$ and hypertension ${ }^{(12)}$. Therefore, we cannot rule out a possibility that weight gain in postmenopausal women may be one of the reasons for weaker associations between $25(\mathrm{OH}) \mathrm{D}$ and blood pressure in postmenopausal women compared with premenopausal women.

When we controlled for physical conditions (height, weight, diabetes, hormone therapy and use of birth-control pills), our observed association between total $25(\mathrm{OH}) \mathrm{D}$ or $25(\mathrm{OH}) \mathrm{D}_{3}$ and hypertension became less significant (see online supplementary material, Supplemental Table S3). Specifically, we confirmed from sensitivity analysis that lowered significance was caused by adding the weight covariate to the model (data not shown). Those observations accord with previous studies, namely that adjusting for BMI results in a lower association between 25(OH)D intake and blood pressure ${ }^{(41,42)}$. Those studies explained, however, that it is still unclear whether weight is a confounder or an intermediary in the possible causal pathway between 25(OH)D and blood pressure. Also, we considered an inflammatory marker known to be associated with hypertension $^{(43)}$ as a potential confounder. When we controlled for serum C-reactive protein concentration in statistical models, the associations between $25(\mathrm{OH}) \mathrm{D}$ and hypertension became less significant but consistent with the results without adjustment for C-reactive protein concentration (see online supplementary material, Supplemental Table S4).

We further examined associations between 25(OH)D and hypertension after controlling for outdoor activity, the major pathway to achieve vitamin $\mathrm{D}$ through sun exposure; however, controlling for outdoor activity did 
not alter those associations. There is the possibility that measuring outdoor activity may mainly capture physical exercise, which itself may also reduce the risk of hypertension. Therefore we cannot rule out misclassification of physical exercise and its residual confounding of outdoor activity.

One might argue that our observed associations could be caused by age difference, as suggested by Maas and Franke $^{(21)}$, because menopausal status is so highly correlated with women's age. In order to determine that our observations were due to menopausal status and not to age, we would need to compare the results in premenopausal $v$. postmenopausal women among a subset of young women ( $<55$ years of age) and among a subset of old women ( $\geq 55$ years of age). We did not conduct the analyses, however, because the number of postmenopausal women in a subset of women $<55$ years of age and the number of premenopausal women in a subset of women $\geq 55$ years of age were too small for a reliable statistical analysis. Instead, we conducted another sensitivity analysis of $25(\mathrm{OH}) \mathrm{D}$ concentrations and risk of hypertension in men, and no significant difference was observed in young compared with old men (data not shown).

We cannot rule out the possibility that associations accorded to menopausal status may be due to differences in personal characteristics. We therefore examined interaction models by all characteristics listed above and were able to confirm that associations between serum 25(OH)D and hypertension did not depend on those factors, varying only in regard to diabetes mellitus (data not shown).

Major strengths of the present study include our use of data from a representative sample of US women, which supports the increased power for our findings. Additionally, we adjusted our models for a variety of potential confounding factors including various dietary intakes and medical treatment, and our results persisted after adjustment for those factors. Our study used serum $25(\mathrm{OH}) \mathrm{D}$ as an exposure biomarker that is able to capture both vitamin $\mathrm{D}$ from outdoor activities and vitamin $\mathrm{D}$ via food and/or supplement intake. Nevertheless, the present study has several limitations. First, because of the study's cross-sectional design, we cannot demonstrate causality. Second, although we controlled for a variety of potential confounders there is still the possibility of residual confounding. Because there were no data available in NHANES 2007-2010, we did not control for serum PTH as a covariate, although PTH, a calciotropic hormone, is suggested as a mediator in the association between vitamin $\mathrm{D}$ and hypertension in which a decrease in serum vitamin D leads to a rise in serum PTH, and elevated PTH has been linked to hypertension ${ }^{(42)}$. Finally, although we used a commonly used biomarker to estimate vitamin D status, our measures from total $25(\mathrm{OH}) \mathrm{D}$ and $25(\mathrm{OH}) \mathrm{D}_{3}$ may not be able to capture bioavailable vitamin $\mathrm{D}$ body stores.
A recent study by $\mathrm{Yu}$ et al. suggests that bioavailable and free $25(\mathrm{OH}) \mathrm{D}$ are emerging measurements of vitamin $\mathrm{D}$ and reports that serum bioavailable and free $25(\mathrm{OH}) \mathrm{D}$ rather than total $25(\mathrm{OH}) \mathrm{D}$ level may be useful in prediction for all-cause and cardiovascular mortality ${ }^{(44)}$. Because there were no data available in NHANES, we did not investigate the associations of bioavailable and free 25(OH)D with the risk of hypertension.

\section{Conclusion}

In conclusion, the present study suggests a significant association between $25(\mathrm{OH}) \mathrm{D}$ status and hypertension in premenopausal women. Women are likely to have vitamin D deficiency because of their negative attitudes towards outdoor activities, including fair skin preference, and indoororiented lifestyle ${ }^{(45,46)}$. Our findings suggest that at least moderate vitamin D status could effectively reduce the risk of hypertension in premenopausal women. In addition to outdoor activities in sunshine (20-30 min of daytime, 2-3 times weekly) ${ }^{(47)}$, a diet rich in vitamin D (oily fish such as salmon, sardines and mackerel, egg yolks, sun-dried mushrooms) and over-the-counter supplementation ${ }^{(7)}$ may contribute to a reduced risk of hypertension in premenopausal women.

\section{Acknowledgments}

Acknowledgments: This study analysed data provided by the National Health and Nutrition Examination Survey 2007-2010. Financial support: This work was supported by the Basic Science Research Program through the National Research Foundation of Korea (NRF) funded by the Korea Ministry of Education (grant numbers 2013R1A6A3A04059556 and 2017R1A6A3A11034504) and the Korea Ministry of Science and ICT (grant number 2015R1C1A2A01054768). Conflict of interest: None of the authors reported a conflict of interest. Authorship: J.H.K. and Y.-H.C. designed and created the study concept; J.H.K. acquired the data, performed the statistical analysis and wrote the paper; Y.-C.H. and Y.-H.C. contributed critical advice and revisions of the manuscript; J.H.K. and Y.-H.C. had responsibility for the entire content of the manuscript and obtained funding; Y.-H.C. supervised the study; and all authors had full access to the study data and read and approved the final manuscript. Ethics of buman subject participation: Not applicable.

\section{Supplementary material}

To view supplementary material for this article, please visit https://doi.org/10.1017/\$1368980019003665 


\section{References}

1. Cheriyan J, O'Shaughnessy KM \& Brown MJ (2010) Primary prevention of CVD: treating hypertension. BMJ Clin Evid 2010, 0214.

2. Zhao G, Ford ES, Li C et al. (2012) Serum 25-hydroxyvitamin $\mathrm{D}$ levels and all-cause and cardiovascular disease mortality among US adults with hypertension: the NHANES linked mortality study. J Hypertens 30, 284-289.

3. Kotsis V, Stabouli S, Papakatsika S et al. (2010) Mechanisms of obesity-induced hypertension. Hypertens Res 33, 386-393.

4. Sur G, Sur M, Kudor-Szabadi L et al. (2010) Arterial hypertension - prevalence of risk factors and morbid associations that increase cardiovascular risk. Maedica 5, 34-40.

5. Majid DS, Prieto MC \& Navar LG (2015) Salt-sensitive hypertension: perspectives on intrarenal mechanisms. Curr Hypertens Rev 11, 38-48.

6. Talukder MA, Johnson WM, Varadharaj S et al. (2011) Chronic cigarette smoking causes hypertension, increased oxidative stress, impaired NO bioavailability, endothelial dysfunction, and cardiac remodeling in mice. Am J Physiol Heart Circ Physiol 300, H388-H396.

7. Holick MF (2007) Vitamin D deficiency. N Engl J Med 357, 266-281.

8. Liu ZM, Woo J, Wu SH et al. (2013) The role of vitamin D in blood pressure, endothelial and renal function in postmenopausal women. Nutrients 5, 2590-2610.

9. Li YC, Kong J, Wei M et al. (2002) 1,25-Dihydroxyvitamin $\mathrm{D}_{3}$ is a negative endocrine regulator of the renin-angiotensin system. J Clin Invest 110, 229-238.

10. Qiao G, Kong J, Uskokovic M et al. (2005) Analogs of $1 \alpha, 25$-dihydroxyvitamin $\mathrm{D}_{3}$ as novel inhibitors of renin biosynthesis. J Steroid Biochem Mol Biol 96, 59-66.

11. Tukaj S, Trzonkowski P \& Tukaj C (2012) Regulatory effects of 1,25-dihydroxyvitamin $\mathrm{D}_{3}$ on vascular smooth muscle cells. Acta Biochim Pol 59, 395-400.

12. Ke L, Mason RS, Kariuki M et al. (2015) Vitamin D status and hypertension: a review. Integr Blood Press Control 8, 13-35.

13. Forman JP, Giovannucci E, Holmes MD et al. (2007) Plasma 25-hydroxyvitamin D levels and risk of incident hypertension. Hypertension 49, 1063-1069.

14. Griffin FC, Gadegbeku CA \& Sowers MR (2011) Vitamin D and subsequent systolic hypertension among women. Am J Hypertens 24, 316-321.

15. Margolis KL, Martin LW, Ray RM et al. (2012) A prospective study of serum 25-hydroxyvitamin D levels, blood pressure, and incident hypertension in postmenopausal women. Am J Epidemiol 175, 22-32.

16. Wang L, Ma J, Manson JE et al. (2013) A prospective study of plasma vitamin D metabolites, vitamin D receptor gene polymorphisms, and risk of hypertension in men. Eur J Nutr $\mathbf{5 2}$, 1771-1779.

17. Ke L, Graubard BI, Albanes D et al. (2013) Hypertension, pulse, and other cardiovascular risk factors and vitamin D status in Finnish men. Am J Hypertens 26, 951-956.

18. Freeman EW, Sammel MD, Lin H et al. (2010) Obesity and reproductive hormone levels in the transition to menopause. Menopause 17, 718-726.

19. Bitoska I, Krstevska B, Milenkovic T et al. (2016) Effects of hormone replacement therapy on insulin resistance in postmenopausal diabetic women. Open Access Maced J Med Sci 4, 83-88.

20. Coylewright M, Reckelhoff JF \& Ouyang P (2008) Menopause and hypertension: an age-old debate. Hypertension 51, 952-959.

21. Maas AH \& Franke HR (2009) Women's health in menopause with a focus on hypertension. Neth Heart J 17, 68-72.
22. Centers for Disease Control and Prevention (2018) National Health and Nutrition Examination Survey 2007-2008. Data, Documentation, Codebooks, SAS Code. https://wwwn.cdc. gov/nchs/nhanes/continuousnhanes/default.aspx?Begin Year=2007 (accessed December 2019).

23. Centers for Disease Control and Prevention (2018) National Health and Nutrition Examination Survey 2009-2010. Data, Documentation, Codebooks, SAS Code. https://wwwn.cdc. gov/nchs/nhanes/continuousnhanes/default.aspx?Begin Year=2009 (accessed December 2019).

24. Centers for Disease Control and Prevention (2010) National Health and Nutrition Examination Survey 2007-2008. Laboratory Procedure Manual: 25-Hydroxyvitamin D3, C3-epi-25-Hydroxyvitamin D3, and 25-Hydroxyvitamin D2 in serum by Ultra High Performance Liquid Chromatographytandem Mass Spectrometry. http://www.cdc.gov/nchs/data/ nhanes/nhanes_07_08/VID_E_met_Vitamin_D.pdf (accessed November 2017).

25. Centers for Disease Control and Prevention (2010) National Health and Nutrition Examination Survey 2009-2010. Laboratory Procedure Manual: 25-hydroxyvitamin D3, C3-epi-25-Hydroxyvitamin D3, and 25-Hydroxyvitamin D2 in Serum by Ultra High Performance Liquid Chromatography-tandem Mass Spectrometry. http://www. cdc.gov/nchs/data/nhanes/nhanes_09_10/VID_F_met_ Vitamin_D.pdf (accessed November 2017).

26. Singh RJ, Taylor RL, Reddy GS et al. (2006) C-3 epimers can account for a significant proportion of total circulating 25-hydroxyvitamin D in infants, complicating accurate measurement and interpretation of vitamin D status. J Clin Endocrinol Metab 91, 3055-3061.

27. Tai SS, Bedner M \& Phinney KW (2010) Development of a candidate reference measurement procedure for the determination of 25-hydroxyvitamin $\mathrm{D}_{3}$ and 25-hydroxyvitamin $\mathrm{D}_{2}$ in human serum using isotope-dilution liquid chromatography-tandem mass spectrometry. Anal Chem 82, $1942-1948$.

28. Institute of Medicine, Committee to Review Dietary Reference Intakes for Vitamin D and Calcium (2011) Dietary Reference Intakes for Calcium and Vitamin D [AC Ross, CL Taylor, AL Yaktine et al., editors]. Washington, DC: National Academies Press.

29. Armstrong T \& Bull F (2006) Development of the World Health Organization global physical activity questionnaire (GPAQ). J Public Health 14, 66-70.

30. Holick MF, Binkley NC, Bischoff-Ferrari HA et al. (2011) Evaluation, treatment, and prevention of vitamin $\mathrm{D}$ deficiency: an endocrine society clinical practice guideline. J Clin Endocrinol Metab 96, 1911-1930.

31. Reis J \& Lutsey P (2012) Vitamin D and cardiovascular disease. In Vitamin D: Oxidative Stress, Immunity, and Aging, pp. 363-384 [AF Gombart, editor]. Boca Raton, FL: CRC Press.

32. Saleh FN, Schirmer H, Sundsfjord J et al. (2003) Parathyroid hormone and left ventricular hypertrophy. Eur Heart J 24, 2054-2060.

33. Dong J, Wong SL, Lau CW et al. (2012) Calcitriol protects renovascular function in hypertension by down-regulating angiotensin II type 1 receptors and reducing oxidative stress. Eur Heart J 33, 2980-2990.

34. Beveridge LA, Struthers AD, Khan F et al. (2015) Effect of vitamin D supplementation on blood pressure: a systematic review and meta-analysis incorporating individual patient data. JAMA Intern Med 175, 745-754.

35. Vimaleswaran KS, Cavadino A, Berry DJ et al. (2014) Association of vitamin D status with arterial blood pressure 
and hypertension risk: a mendelian randomisation study. Lancet Diabetes Endocrinol 2, 719-729.

36. Lim HS, Kim TH, Lee HH et al. (2016) Hypertension and age at onset of natural menopause in Korean postmenopausal women: results from the Korea National Health and Nutrition Examination Survey (2008-2013). Maturitas 90, 17-23.

37. Nickenig G, Baumer AT, Grohe C et al. (1998) Estrogen modulates AT1 receptor gene expression in vitro and in vivo. Circulation 97, 2197-2201.

38. Gilad LA, Bresler T, Gnainsky J et al. (2005) Regulation of vitamin $\mathrm{D}$ receptor expression via estrogen-induced activation of the ERK 1/2 signaling pathway in colon and breast cancer cells. J Endocrinol 185, 577-592.

39. Lovejoy JC, Champagne CM, de Jonge L et al. (2008) Increased visceral fat and decreased energy expenditure during the menopausal transition. Int J Obes (Lond) 32, 949-958.

40. Wortsman J, Matsuoka LY, Chen TC et al. (2000) Decreased bioavailability of vitamin D in obesity. Am J Clin Nutr 72, 690-693.

41. Kim H, Chung YE, Jung SC et al. (2013) Independent associations of circulating 25-hydroxyvitamin D and parathyroid hormone concentrations with blood pressure among Koreans: the Korea National Health and Nutrition Examination Survey (KNHANES), 2009-2010. Calcif Tissue Int 93, 549-555.

42. He JL \& Scragg RK (2011) Vitamin D, parathyroid hormone, and blood pressure in the National Health and Nutrition Examination Surveys. Am J Hypertens 24, 911-917.

43. Sesso HD, Wang L, Buring JE et al. (2007) Comparison of interleukin- 6 and C-reactive protein for the risk of developing hypertension in women. Hypertension 49, 304-310.

44. Yu C, Xue H, Wang L et al. (2018) Serum bioavailable and free 25-hydroxyvitamin D levels, but not its total level, are associated with the risk of mortality in patients with coronary artery disease. Circ Res 123, 996-1007.

45. Jang H, Koo FK, Ke L et al. (2013) Culture and sun exposure in immigrant East Asian women living in Australia. Women Health 53, 504-518.

46. Ke L, Mason RS, Mpofu E et al. (2015) Vitamin D and parathyroid hormone status in a representative population living in Macau, China. J Steroid Biochem Mol Biol 148, 261-268.

47. Pearce SH \& Cheetham TD (2010) Diagnosis and management of vitamin D deficiency. BMJ 340, b5664. 